\title{
Issues in Applying Model Based Process Improvement in the Cloud Computing Domain
}

\author{
Jeremy Cade ${ }^{1}$, Lian $\mathrm{Wen}^{2,3}$, and Terry Rout ${ }^{2}$ \\ 1 Machine Intelligence and Pattern Analysis Laboratory (MiPAL), Griffith University \\ ${ }^{2}$ Institute of Integrated and Intelligent Systems (IIIS), Griffith University \\ ${ }^{3}$ School of Information and Communication Technology (ICT), Griffith University \\ 170 Kessels Rd, Qld 4111, Australia \\ jeremy.cade@griffithuni.edu.au, 1.wen@griffith.edu.au, \\ t.rout@griffith.edu.au
}

\begin{abstract}
Cloud Computing offers organisations a range of benefits, both economic and technological. However the decision to deploy an application or service to the cloud is a not a trivial one. Organisations need to be fully aware of not only the business requirements for a given application or service, but also the technological requirements and or constraints of the cloud. Model-based process assessment and improvement has been shown to support organisational change in different domains of application, but there are few reports of application in cloud computing. As a first step in defining suitable models to support process management, the impact of working with cloud resources on existing standard processes has been examined using the techniques of behavior engineering. A path for future work is proposed.
\end{abstract}

Keywords: Process Model, Software Process, Behavior Engineering, Cloud Computing

\section{Introduction}

Organisations of all sizes are trending towards the Cloud Computing model as the preferred means of provisioning new, or migrating existing Information Technology (IT) systems. Cloud Computing offers organisations a greater degree of IT flexibility through access to inexpensive on-demand IT resources that are able to meet high levels of availability, reliability and scalability. This paradigm shifts the responsibility of maintenance and ownership of IT infrastructure and computing services from the organisation to Cloud Computing provides, who in turn make these services available as pay-per-use commodities.

The primary push to adopt Cloud Computing has historically come from organisations such as IBM, Amazon and Microsoft as a way to reduce the cost of IT infrastructure, application development and deployment [14] 25]. A 2013 Forrester Research report found organisations were able to obtain a Risk-Adjusted Return On Investment (ROI) of $349 \%$, along with sizeable improvement in development/test environment setup and configuration by leveraging Microsoft's 
Azure Cloud Computing infrastructure 9. While there are potential economic benefits, software and services engineered specifically for the paradigm are required if organisations wish to take full advantage of the possibilities offered by Cloud Computing.

It has been clearly demonstrated 13 that model-based process improvement provides a firm basis for defining productive approaches to development and operation of IT systems, specifically addressing the needs of particular domains; application to defence, aerospace, automotive and medical device systems have been documented. However, there are few (if any) reports on adaptation of common processes to working with cloud infrastructure. Our aim in this paper is to provide an initial evaluation of the issues and potential for process definition and improvement in relation to cloud computing.

In exploring potential process improvement strategies, it is useful to distinguish between the "basic" systems development processes, dealing with the specific engineering activities involved in developing the system, and the "infrastructure" processes concerned with managing and controlling the system development. The collection of infrastructure processes can commonly be represented by a "quality management system", and it is not unusual for process improvement in different domains to focus on the tailoring of the infrastructure, while leaving the basic processes largely unaffected. This can, however, result in significant problems and inefficiencies if the domain requires modification of the basic processes. The first step in applying process improvement in the cloud computing domain should be to determine whether specific tailoring of the basic processes is required, or whether improvement to the infrastructure processes alone is sufficient.

The set of basic processes are defined in International Standards such as ISO/IEC 12207 and ISO/IEC 15288; these also provide definitions of the bulk of the infrastructure processes. The distinction between the two classes of process is made clearer in process assessment models such as ISO/IEC 15504-5 and related organisational maturity models (ISO/IEC 15504-7).

In this paper we asses the current set of Software Engineering standards in relation to Cloud Computing. The Behavior Engineering technique, which has been demonstrated to be useful in verifying consistency in process definitions is applied to the common Process Reference Model (PRM) of ISO/IEC 12207 and ISO/IEC 15288, specifically looking at the Stakeholder Needs and Requirements Definition Process.

The rest of this paper is organised as follows. Section 2 provides background information on Cloud Computing, Process Management and Improvement, and Behavior Engineering. Section 3 describes the issues with the current Software Engineering approaches and provides a case study. Finally the paper is concluded and we set forth future research in Section 4 . 


\section{Background}

\subsection{Cloud Computing}

The Cloud Computing paradigm is a significant shift from traditional in-house IT infrastructure, allowing organisations to effectively deliver a wide range of services to consumers at lower cost [1. The US Institute of Standards and Technology (NIST) provides the following definition[20]: Cloud computing is a model for enabling ubiquitous, convenient, on-demand network access to a shared pool of configurable computing resources (e.g., networks, servers, storage, applications, and services) that can be rapidly provisioned and released with minimal management effort or service provider interaction.

The Cloud Computing paradigm is comprised of four deployment models and three service models. The deployment models, Community Cloud, Hybrid Cloud, Public Cloud and Private Cloud all provides varying levels of tenancy of the underlying Cloud Computing infrastructure. At one end of the scale, Private Cloud provides resources for a single tenant, it may be provided offsite and managed by a Cloud Computing provider, or on-site and managed in a way similar to traditional IT infrastructure. At the other end of the scale is the Public Cloud, where resources are shared between multiple tenants. Community Cloud is similar to Private in that infrastructure is provisioned for a single community of tenants from multiple organisations that share a common concern. The Hybrid Cloud model is a combination of one or more of the three previous Cloud deployment models. The three service models Software as a Service (SaaS), Platform as a Service (PaaS) and Infrastructure as Service (IaaS) provide varying levels of control and management over the underlying cloud infrastructure. In the SaaS model software is delivered as a service on cloud infrastructure and is provided to a consumer or user through the use of a thin-client (e.g. a web-browser) or an Application Programming Interface (API). SaaS services are often consumer focused, but may also be enterprise services. PaaS provides a development platform and services that assist developers in deploying applications or software in a controlled manner. The developer has no responsibility for the underlying operating system configuration or infrastructure. IaaS capabilities are provided for the virtualisation of entire data-centres, providing Consumers with the ability to control infrastructure from the operating system up.

Cloud Computing providers may offer one of more deployment models, combined with one or more service models as part of a wider product offering, e.g. Both Amazon and Microsoft ${ }^{4}$ offer both Infrastructure as a Service (IaaS) and Platform as a Service (PaaS), where as Google only provides a PaaS solution ${ }^{5}$.

Previous research 6] 11, 10, 23, 29] in the Cloud Computing domain have identified a range of Software Engineering issues, though all agree that Requirements Engineering (RE) to be of significant importance.

RE has said to be the single hardest part of building a software system 4 . Requirements are often poorly defined in ambiguous language or exist within a

\footnotetext{
${ }^{4}$ Microsoft Azure: http://azure.microsoft.com/en-us/

${ }^{5}$ https://developers.google.com/appengine/training/intro/whatisgae\#paas
} 
large problem space. The traditional RE approaches can be broken down into five broad groups (Elicitation, Modelling, Requirements Analysis, Validation \& Verification and Requirements Management) [5]. The traditional RE approach focuses on the elicitation and verification of requirements from the users and organisations that will make use of a software system. The different deployment and service models all have a slightly different set of requirements, particularly in relation to the required Software Engineering and/or developer skill sets 22. These requirements may or may not be communicated by a Cloud Computing. Additional non-functional requirements also need to be considered by those tasked with RE when considering Cloud Computing deployments. These include:

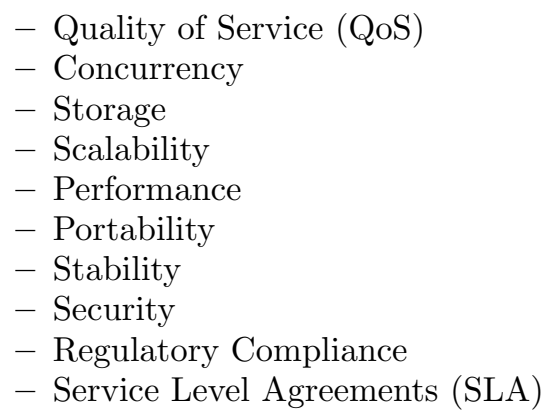

Cloud Computing providers, as a supplier of infrastructure also need to be aware of an organisations requirements. This somewhat complicates the process of selecting acceptable Cloud Computing providers.

Service Management is of critical importance to successful implementation of Cloud Computing deployments. However there are a number of areas that may be affected by either the choice of Cloud Computing deployment model or the proposed workload. For example; A data and application based workload such as data-mining may be better suited to a private cloud, which may present additional service management requirements in relation to the cost of the infrastructure and organisation expertise deployment. IBM has suggested that Service Management Maturity Level Four processes are likely needed in order for a Cloud Computing deployment to be successful[15].

\subsection{Process Management and Improvement}

In addressing concerns with the deployment of a new infrastructure such as Cloud Computing, process management and improvement approaches offer much that is of benefit. As demonstrated by Humphrey [13], traditional approaches of process improvement can have significant impact in the IT environment. Following the model of ISO/IEC 24774, processes can be described in terms of their purpose and the outcomes of implementation; these descriptions can then be used as the basis for identifying strengths, weaknesses and improvement opportunities in the operations of the processes.

In order to apply this approach to Cloud Computing, the nature of the processes involved in working in a Cloud environment needs to be examined in more 
depth. A starting point for this can be found in the international standards for software and system life cycle processes, ISO/IEC 12207 and ISO/IEC 15288. These two core standards are currently under revision, and the new releases will be based around a set of core processes common to both standards. Following the framework of ISO/IEC 33001 and ISO/IEC 33004, we can say that the two standards have a common Process Reference Model (PRM), and we can extract process definitions generally applicable for software and systems development from this PRM.

As noted above, a key issue in Cloud Computing is the issues associated with Requirements Engineering. In the common PRM, the relevant process is the Stakeholder Needs and Requirements Definition process, defined as follows:

Purpose: The purpose of the Stakeholder Needs and Requirements Definition process is to define the stakeholder requirements for a system that can provide the capabilities needed by users and other stakeholders in a defined environment.

It identifies stakeholders, or stakeholder classes, involved with the system throughout its life cycle, and their needs. It analyses and transforms these needs into a common set of stakeholder requirements that express the intended interaction the system will have with its operational environment and that are the reference against which each resulting operational capability is validated. The stakeholder requirements are defined considering the context of the system of interest with the interoperating systems and enabling systems.

Outcomes: As a result of the successful implementation of the Stakeholder Needs and Requirements Definition process:

a) Stakeholders of the system are identified.

b) Required characteristics and context of use of capabilities and concepts in the life cycle stages, including operational concepts, are defined.

c) Constraints on a system are identified.

d) Stakeholder needs are defined.

e) Stakeholder needs are prioritised and transformed into clearly defined stakeholder requirements.

f) Critical performance measures are defined.

g) Stakeholder agreement that their needs and expectations are reflected adequately in the requirements is achieved.

h) Inputs for requirements of any enabling systems or system elements that serve the stakeholder needs and requirements activities are identified.

i) Any enabling systems or services needed for stakeholder needs and requirements are available.

j) Traceability of stakeholder requirements to stakeholders and their needs is established. 


\subsection{Behavior Engineering}

Initially described in 2003, Behavior Engineering (BE) [8] [ ] [3] is a formalised process allowing Software Engineers to translate natural language software requirements into a complete and consistent requirements specifications [3. Behavior Engineering consists of the Behavior Modelling Process (BMP) and the Behavior Modelling Language (BML) notation [fig. 1], a formally grounded graphical notation consisting of Behavior Trees (BT) and Component or Composition Trees (CT) [3].

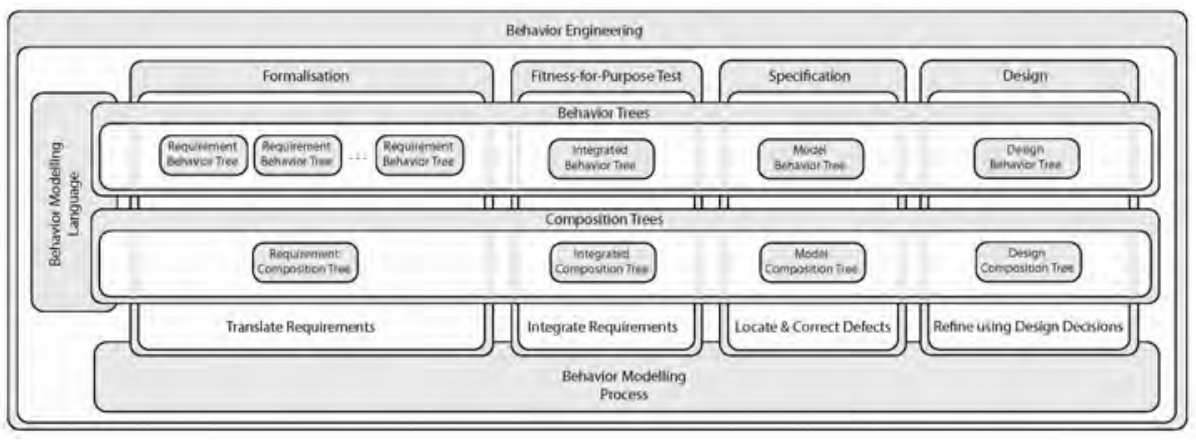

Fig. 1. Overview of Behavior Engineering

\subsection{Behavior Modelling Process}

The Behavior Modelling Process (BMP) consists of four distinct stages, Formalisation, Fitness for Purpose Test, Specification and Design. At each stage the process makes use of BML to address problems of scale, complexity and ambiguous language within natural language requirements.

Formalisation requirements are modelled one a time to for a Requirement Behavior Tree (RBT) and a corresponding Requirements Composition Tree (CBT) whilst trying to capture the original intent of the natural language requirement while removing ambiguity.

Fitness for Purpose RBTs are integrated together to form a Integrated Behavior Tree (IBT), while CBTs are integrated into Integrated Composition Tree (ICT) further reducing the number of errors and ambiguity in the natural language requirements.

Specification an executable specification called a Model Behavior Tree (MBT) is created by addressing the issues of incompleteness, inconsistency and redundancy within the IBT.

Design system boundary decisions are applied to the MBT 


\subsection{Behavior Modelling Language}

As mentioned above the Behavior Modelling Language is a formally-grounded graphical notation.

BT are a formal, tree-like graphical form that represents behaviour of individual or networks of entities which realise or change states, make decisions, respond-to/cause events, and interact by exchanging information and/or passing control 8 .

$\mathrm{CT}$ contain the complete system vocabulary, which by definition is consistent with the vocabulary used in BT as they both originate from the same natural language requirements. CT are a tree of components arranged into a compositional hierarchy using structural and functional aggregation or specialisation relations. Each component in the $\mathrm{CT}$ contains the complete set of states, attributes, events and relations in which the component is responsible for. CT are an important tool in resolving defects not visible in individual Requirement Behavior Trees, such as aliases.

Previous research has shown that aspects of BE may be of use in a wide range of applications including:

- Integration of software \& hardware modelling [19]

- Requirements analysis in large scale projects 7] 21

- Risk-based testing 28

- Semi-automated hazard analysis 12

- Process modelling, comparison and validation [24] 26] 27]

\subsection{Using Composition Trees to Model Software Processes}

According to ISO/IEC 24774:2007 [18, the standard elements to describe a process include the title, the purpose, outcomes, activities and tasks. Apart from the title, which is only the name of a process, purpose and outcomes are more static elements, so they may be more suitable to be modelled by composition trees.

The benefit to model process in a composition tree is that the graph presentation provides a overall view of the process. The graphic presentation is less ambiguous, and formal verification such as comparing two processes can be performed by using automated tools [26.

Details about how to construct a Composition Tree from a process's purpose and/or outcomes can be found in our previous papers 26] 27. Here we only use one simple example to illustrate the composition tree constructed from the purpose of Configuration Management process defined in ISO/IEC 12207, AMD $1 / 2,2000$ [16].

Process Name Configuration management.

Process Purpose The purpose of the Configuration management process is to establish and maintain the integrity of the work products/items of a process or project and make them available to concerned parties. 
In order to model this process, the first step is to identify and list all the components:

CMP Configuration Management Process

WPI Work product or work item

CPT Concerned Party

The composition tree translated from the process purpose is presented in fig. 2. which shows that there are two different types of components under CMP, WPI and CPT; the ${ }^{*}$ sign indicates that the component may have more than one instance. The WPI has an attribute called integrity and the integrity needs to be established and maintained. There is also a relationship for WPI; the relationship is that WPIs should be available to CPTs. The tag $\mathrm{P}$ in each box means this piece of compositional information is translated from the purpose of the process.

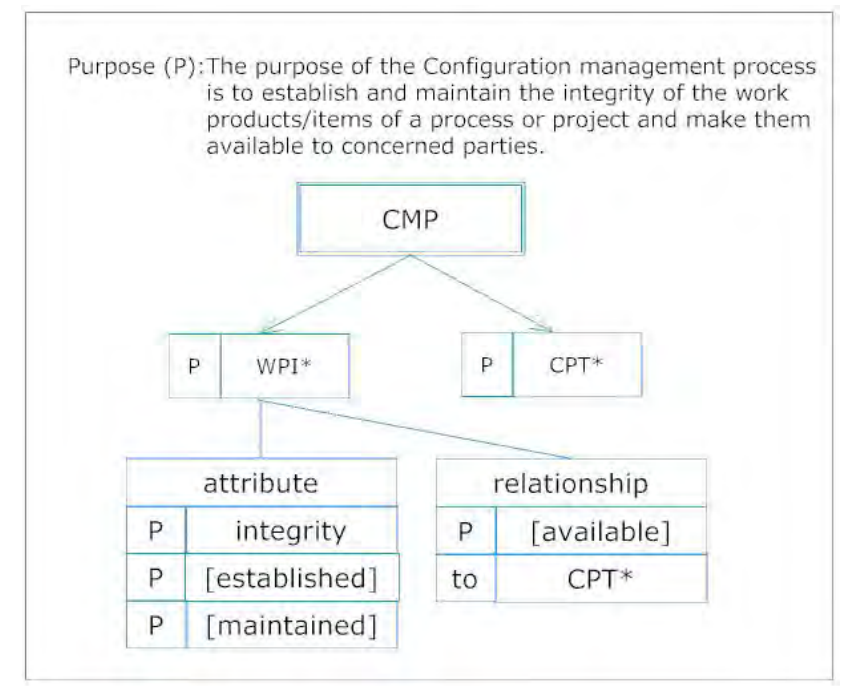

Fig. 2. The CT constructed from the purpose of the Configuration Management process

\section{A Case Study}

As identified, the impact from Cloud Computing on Software Engineering is alldimensional and significant [10, ranging from requirement definition, requirement analysis, design and implementation to testing, deployment and maintenance. Therefore, in order to provide cloud computing based high quality software systems economically, the entire spectrum of software processes in the software life-cycle might need to be reviewed to address the impact from the cloud computing. 
In this paper, we examine the Stakeholder Need and Requirement Definition Process defined in the latest version of Systems and software engineering - software life cycle process [17]. All the outcomes of this process are listed in the previous section; here we modelled the outcomes in a composition tree [fig. 3. In this composition tree, CPM stands for critical performance measure and ESSE means enabling system or system element.

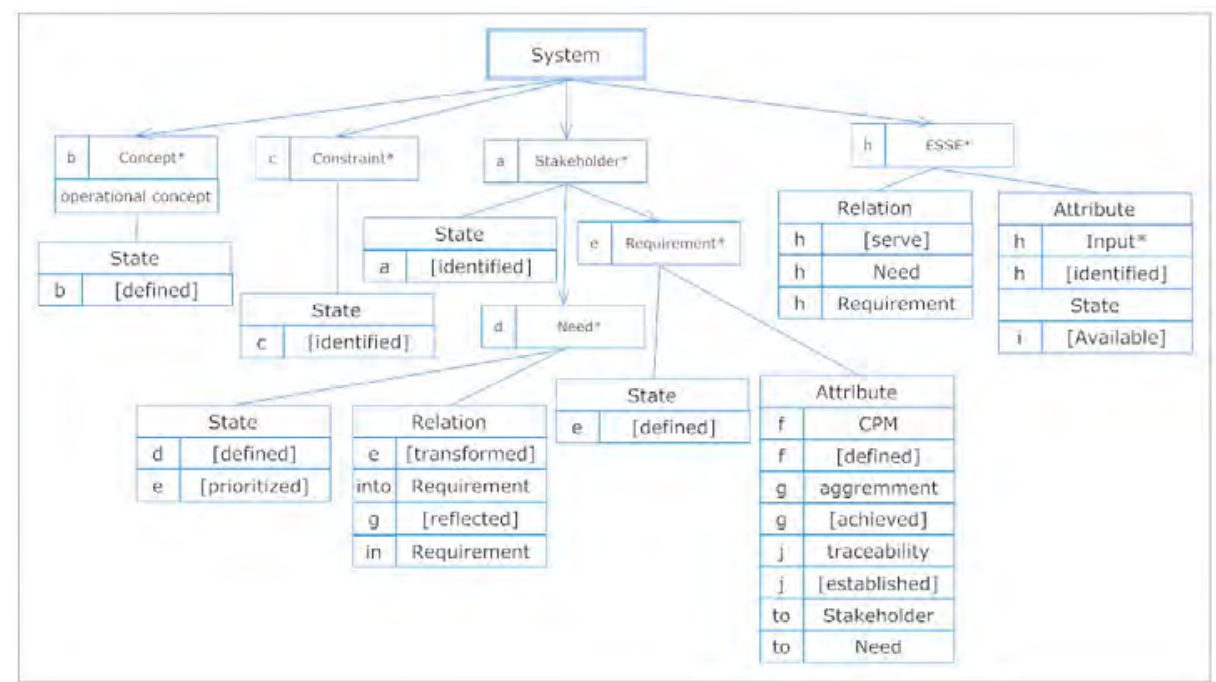

Fig. 3. Stakeholder Needs and Requirements Definition, modelled as a Composition Tree

The difference between a traditional software project and a software project with Cloud Computing involved is that the later introduces an important stakeholder, the Cloud Computing service provider, who has dramatic influence on the project during the entire project life cycle. As this paper focus on the requirement definition stage, we only discuss the impact from the cloud service provider on the requirement definition stage.

For Cloud Computing enabled software systems, they usually confront following issues. Firstly, as a Cloud Computing enabled software system will have its data and services hosted and maintained in a third party Cloud Computing environment, security is always a concern, though is often demonstrated, many organisations are not knowledgeable of their own security needs nor they can state their security requirements properly [2]. Secondly, different Cloud Computing service providers might have different delivery capabilities. To match the customer's needs to the service provided by different Cloud Computing service providers and identify the most suitable one is not a trivial task [10. Thirdly, due to the capability of the Cloud Computing service provider and budget, the 
customer needs to negotiate the requirements with the Cloud Computing service provider 29 and sometimes sacrifice the customer's requirements or policy. To illustrate those issues, we will introduce a real case study, the Griffith University Staff Email system, which had encountered a number of the issues.

Griffith University has a comprehensive on-line system called Griffith Portal, which integrates many subsystems covering nearly all the management aspects in the university. The university policy is that there should be only one authentication server for valid users to access all the different subsystems, and the authentication server must be hosted and fully controlled by the university. The university had evaluated a few cloud service providers including Microsoft and Google and finally selected Google to provide the staff email system. However, Google has its own account authentication server and its own privacy policy, which is inconsistent to with Griffith University's privacy policy. Under this situation, Griffith University and Google had to negotiate in order to find a solution that was acceptable for both parties. Eventually, a dedicated adapter is introduced to solve the policy conflicts. Under this arrangement, a user may feel that he/she is still using the ID and password assigned with their University account to login into the email service, however a second secret password is generated in the back-end to satisfy the authentication requirements of a Google account. This adapted solution is made slightly more transparent when a user tires to use a mobile device to access the email account. The user has to retrieve the second secret password and use it on the mobile device directly.

\section{Conclusions \& Future Research Topics}

The case study above indicates that for a cloud involved software system, some important practices such as evaluating different cloud service providers, finding out the best match service provider, negotiating with the service provider regarding to the requirements, adjusting and even sacrificing some requirements are critical to the success of the system. However, those practices and their expected outcomes have not been adequately addressed in the traditional software process. At least the current stakeholder needs and requirement definition process does not explicitly state them in its outcomes. People may argue that even though the process outcomes do not address those issues directly, the given outcomes are actually abstract and high-level enough to cover nearly every practices discussed above. However, as those practices are so critical, they should be addressed explicitly to maximum the chance for a project to succeed.

At this stage the research is still in its preliminary stages. Future research will look to complete empirical studies within industry to confirm our initial findings while aiming to specify in detail the basic process model for the Cloud Computing domain. Long term research will be aimed at developing a suite of standards and processes for improving process models within the Cloud Computing domain; it is clear that the results of this research will impact on standards specific to the Cloud Computing domain, within the scope of JTC1 SC38/WG3, as well 
as to the work of WG10 and WG7 in JTC1 SC7, dealing with the definition, assessment and improvement of relevant processes.

\section{References}

1. Armbrust, M., Fox, A., Griffith, R., Joseph, A., Katz, R., Konwinski, A. \& Zaharia, M.: A view of cloud computing. Communications of the ACM, 53(4). pp 50-58. (2010) doi:10.1145/1721654.1721672

2. Beckers, K., Heisel, M.: Structured Pattern-Based Security Requirements Elicitation for Clouds. Eighth International Conference on Availability, Reliability and Security (ARES), pp. 465-474. (2013)

3. Behavior Engineering Web Site, http://www.beworld.org/BE/

4. Brooks, F.: No Silver Bullet: Essence and Accidents of Software Engineering. IEEE Computer, 20(4). pp. 10-19. (1987)

5. Cheng, B.H.C., Atlee, J.M.: Research Directions in Requirements Engineering. Future of Software Engineering (FOSE'07). pp. 285-303. (2007)

6. da Silva, E.A.N., Lucredio, D.: Software Engineering for the Cloud: a Research Roadmap. 26th Brazilian Symposium on Software Engineering (SBES). pp. 71-78 (2012) doi: 10.1109/SBES.2012.12

7. Dromey, R.G.: Climbing Over the 'No Silver Bullet' Brick Wall. IEEE Software 23(2). pp. 118-120 (2006)

8. Dromey, R.G: From Requirements to Design: Formalising the Key Steps. IEEE International Conference on Software Engineering and Formal Methods (SEFM'03). pp. 2-11 (2003)

9. Forrester Research : The Total Economic Impact of Windows Azure. (2013). Retrieved from http://info.windowsazure.com/rs/microsoft/images/WhitePaper _ The_Total_Economic_Impact_Of_Microsoft_Windows_Azure_Forrester.pdf

10. Guha, R., Al-Dabass, D.: Impact of Web 2.0 and Cloud Computing Platform on Software Engineering. 2010 International Symposium on Electronic System Design. pp. 213-218. (2010) doi: 10.1109/ISED.2010.48

11. Grundy, J., Kaefer, K., Keong, J., \& Liu, A.: Software Engineering for the Cloud. IEEE Software 29(2). pp. 23-29 (2012)

12. Grunske, L.: An Automated Failure Mode and Effect Analysis based on High-Level Design Specification with Behavior Trees. Proceedings of International Conference on Integrated Formal Methods (IFM). pp. 129-149 (2005)

13. Humphrey, W.S.: Managing the Software Process (Hardcover). Addison-Wesley Professional. (1989)

14. IBM : IBM Perspective on Cloud Computing. (2008). Retrieved from ftp://ftp.software.ibm.com/software/tivoli/brochures/IBM_Perspective_ on_Cloud_Computing.pdf

15. IBM : Integrated service management and cloud computing. (2010). Retrieved from http://www.ibm.com/ibm/files/E955200R99025N70/5Integrated_service_ management_and_cloud_computing_644KB.pdf

16. ISO/IEC 12207:1988, AMD 1 / 2:2000 Information technology Software engineering \& Software life cycle processes (2000)

17. ISO/IEC IEEE 12207 CD1 - revision of 12207:2008 Systems and software engineering Software life cycle processes (2014)

18. ISO/IEC TR 24774. Software and systems engineering - Life cycle management Guidelines for process description.(2007) 
19. Myers, T., Fristzon, P., Dromey, R.G.: Seamlessly Integrating Software \& Hardware Modelling for Large-Scale Systems. 2nd International Workshop on Equation-Based Object-Oriented. pp. 5-15 (2008)

20. National Institute of Standards and Technology : The NIST Definition of Cloud Computing (2011)

21. Powell, D.: Requirements evaluations using Behavior Trees - findings from industry. Australian Software Engineering Conference (ASWEC'07). (2007)

22. Ranger, S.: How cloud computing changes (almost) everything about the skills you need. ZDNet (2013). Retrieved from http://www.zdnet.com/ how-cloud-computing-changes-almost-everything-about-the-skills-you-need-7000020163/

23. Rimal, B.P., Jukan, A.., Katsaros, D., Goeleven, Y.: Architectural Requirements for Cloud Computing Systems: An Enterprise Cloud Approach. Journal of Grid Computing 9(1). pp. 3-26 (2012)

24. Tuffley, D., Rout, T.: Behavior Engineering as a Process Model Verification Tool. In: Proceedings of the 10th International SPICE Conference (2010)

25. Varia, J., Mathew, S.: Overview of Amazon Web Services. Amazon (2014). Retrieved from http://media.amazonwebservices.com/AWS_Overview.pdf

26. Wen, L., Tuffley, D., Rout, T.: Using Composition Trees to Model and Compare Software Process. Software Process Improvement and Capability Determination SPICE (2011)

27. Wen, L. Rout, T.: Using Composition Trees to Validate an Entry Profile of Software Engineering Lifecycle Profiles for Very Small Entities (VSEs).Software Process Improvement and Capability Determination SPICE. pp. 38-50 (2012)

28. Wendland, M., Kranz, M., \& Schienferdecker, I.: A Systematic Approach to RiskBased Testing Using Risk-annotated Requirements Models. CSEA 2012 : The Seventh International Conference on Software Engineering Advances. pp. 636-642 (2012)

29. Zardari, S., Bahsoon, R.: Cloud Adoption: A Goal-Oriented Requirements Engineering Approach. Proceedings of the 2nd International Workshop on Software Engineering for Cloud Computing. pp. 29-35. (2011) 